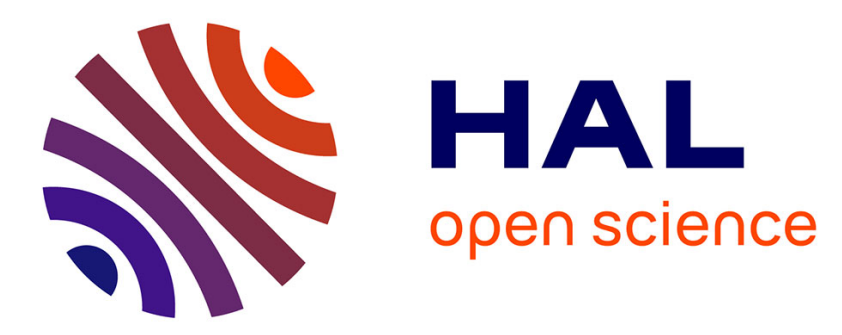

\title{
Quasi-two-dimensional foam flow through and around a permeable obstacle
}

Natalia Shmakova, Thibaud Chevalier, Antti Puisto, Mikko Alava, Christophe Raufaste, Stéphane Santucci

\section{- To cite this version:}

Natalia Shmakova, Thibaud Chevalier, Antti Puisto, Mikko Alava, Christophe Raufaste, et al.. Quasitwo-dimensional foam flow through and around a permeable obstacle. Physical Review Fluids, 2020, 5 (9), 10.1103/PhysRevFluids.5.093301 . hal-03450540

\section{HAL Id: hal-03450540 \\ https://hal.science/hal-03450540}

Submitted on 26 Nov 2021

HAL is a multi-disciplinary open access archive for the deposit and dissemination of scientific research documents, whether they are published or not. The documents may come from teaching and research institutions in France or abroad, or from public or private research centers.
L'archive ouverte pluridisciplinaire HAL, est destinée au dépôt et à la diffusion de documents scientifiques de niveau recherche, publiés ou non, émanant des établissements d'enseignement et de recherche français ou étrangers, des laboratoires publics ou privés. 


\title{
Quasi-two-dimensional foam flow through and around a permeable obstacle
}

\author{
Natalia Shmakova $\odot$ \\ Lavrentyev Institute of Hydrodynamics, Siberian Branch of the Russian Academy of Sciences, \\ Novosibirsk, Russia \\ Thibaud Chevalier $\odot$, Antti Puisto, and Mikko Alava \\ COMP Center of Excellence, Department of Applied Physics, Aalto University, Espoo, Finland \\ Christophe Raufaste \\ Université Côte d'Azur, CNRS, Institut de Physique de Nice, Nice, France \\ Stéphane Santucci* \\ Université de Lyon, ENSL, UCBL, CNRS, Laboratoire de Physique, Lyon, France \\ and Lavrentyev Institute of Hydrodynamics, Siberian Branch of the Russian Academy of Sciences, \\ Novosibirsk, Russia
}

(Received 27 January 2020; accepted 27 July 2020; published xxxxxxxxxx)

3

4

5

We present an experimental study of a two-dimensional liquid foam, composed of a confined monolayer of bubbles, forced to flow within a model porous medium that mimics an inhomogeneous open fracture. It consists of a Hele-Shaw cell with a single localized constriction-like defect that reduces locally its gap and thus its permeability. Taking advantage of the possibility to directly visualize and follow the bubbles, we compute the bubble velocity field by image correlation analysis, as well as the bubble deformation field, through eccentricity measurements obtained by fitting each bubble with an ellipse. The defect acting as a permeable obstacle can strongly disturb the foam flow; we investigate here the influence of its geometry (height, size, and shape) on the average steady-state flow of foams of various liquid content, and specifically the motion and deformation of their elementary components, the bubbles. In the frame of the flowing foam, we can observe a recirculation around the obstacle, characterized by a multipolar velocity field. Its complex structure displays a strong fore-aft asymmetry, with an extended region downstream the constriction, where the foam velocity can be much larger than the imposed driving one. This overshoot was already revealed for nonpermeable obstacles, but here we show that its extent and intensity are associated to the bubble deformation and depend strongly and nontrivially on both the geometry of the constriction as well as the liquid fraction of the foam.

DOI: 10.1103/PhysRevFluids.00.003300

\section{INTRODUCTION}

Liquid foams are dispersed gaseous bubbles within a liquid phase [1]. Despite such a simple composition, they display very specific rheological properties related to their jammed disordered multiscale structure. Indeed, they behave as solids at rest, while they flow as liquids above a critical yield stress [2,3]. Such a dual mechanical behavior is at the root of their use in numerous applications

\footnotetext{
*stephane.santucci@ens-lyon.fr
} 
from everyday life products developed in the food and cosmetic industries to even larger-scale industrial processes, that are, for instance, soil remediation, mineral extraction, or blast mitigation [4].

Foam flows in porous media have been studied for years and still represent a subject of intense research in the context of enhanced oil recovery [5]. Indeed, foam injection into a reservoir could improve the effectiveness of oil recovery processes [6,7] by suppressing viscous fingering instabilities and mitigating sweep inhomogeneities due to permeability variations and gravity override [8-11].

From a more fundamental perspective, two-dimensional foam flows with bubbles confined as a single monolayer have been considered in order to investigate and probe the global rheological behavior of liquid foams and specifically their viscous, elastic, and plastic properties. The twodimensional geometry allows a direct visualization of the evolving foam structure during its flow. For instance, the flow around an obstacle also classically referred as Stokes flow has been studied in full detail [12-15]. Remarkably, such two-dimensional foam flows can be quantitatively described within the framework of continuum mechanics [16].

Nevertheless, discrete effects appear as soon as the bubble size becomes comparable to the obstacle dimensions or to the interobstacles distances [17,18]. Indeed, the impregnation of liquid foams inside a porous structure can exhibit specific properties due to the discrete nature of its elementary components, i.e., the bubbles. In very confined geometries with bubbles larger than the typical pore size, the foam structure corresponds to trains of soap films, lamellae. In constricted pore channels, the pressure drop across the lamellae, which can take asymmetric shapes even in idealized symmetric pores, brings forward a capillary resistance to the flow. This leads to a minimum of pressure gradient required to mobilize a "bamboolike" structure [19-21] at the origin of the effective yield stress of foams in porous media. Moreover, in such confined geometries, bubbles can easily coalesce or divide with direct consequences on the bubble size distribution and foam mobility [22-24].

In the present study, we aim to investigate an intermediate situation where a monolayer of bubbles confined between two glass plates is forced to flow toward a permeable obstacle. The originality of the present study relies on the fact that the obstacle does not fill the gap of the confining medium. This degree of freedom leaves the possibility for the bubbles to go through or above the defect at the expense of strong deformations that can affect the surrounding overall foam flow.

In the following, we present a systematic experimental study, investigating how the average steady-state velocity and bubble deformation fields of a liquid foam invading a confining porous medium are affected by a local permeable defect. We perform experiments with different obstacles, varying notably their geometrical properties (size, shape, and height), but also changing the liquid fraction of the invading liquid foam.

The experimental procedure and data analyzing methods are described in Sec. II. On one hand, the average steady-state velocity field is measured by analyzing spatial correlations of the evolving structure of the foam between subsequent recorded images. On the other hand, the average steadystate bubble deformation field is obtained by investigating the shape of each labeled bubbles of the flowing foam, and, more specifically, by measuring their eccentricity, considering them as ellipses. Moreover, in Sec. II B 3, we define a permeability factor that quantifies the ratio of the flow rate passing through the constriction with respect to the flow rate in absence of defect. We observe a linear trend between this factor and the gap contrast.

The localized constriction defect can strongly disturb the foam flow leading to multipolar and fore-aft asymmetric bubble deformation and velocity fields in the frame of the flowing foam. In particular, we can observe an extended region downstream the constriction, where the bubbles are strongly elongated in the mean direction of the flow and where the foam velocity can be much larger than the imposed driving velocity.

We show in Sec. III A that as the gap contrast between the main cell and the constriction grows, the amplitude of the perturbation becomes larger, with nevertheless a saturation of both the maximum bubble deformation and the velocity overshoot for very strong confinement. We also 
show that the spatial extent of the perturbation is related to the size of the defect as a first-order approximation, while it depends slightly on its shape. Finally, in Sec. III B we report the systematic decrease of the amplitude and extent of both the bubble deformation and the velocity overshoot with an increasing liquid fraction of the foam.

Therefore, as discussed in the last conclusive section, our flow experiments confirm that the elastoplastic properties of the liquid foam — controlled by its liquid fraction—are responsible of this symmetry breaking of the flow fields (deformation and velocity) and, in particular, the overshoot downstream the constriction.

\section{MATERIALS AND METHODS}

\section{A. Setup}

\section{A simple model fracture}

To model the simplest nonhomogeneous open fracture medium, we use a Hele-Shaw cell, consisting of two glass plates, $500 \mathrm{~mm}$ long, $150 \mathrm{~mm}$ wide, and $10 \mathrm{~mm}$ thick, separated by a gap of $G=1.2 \mathrm{~mm}$. This gap is ensured by spacers placed on the side of the cell, together with a sealing toric joint, that sets the effective width of the cell to $130 \mathrm{~mm}$. A single obstacle of either square or circular shape, different lateral size or diameter $(a=10,20$ or $30 \mathrm{~mm})$, and various heights $H$ of typically a few hundred of microns, smaller than the cell gap $H<G$, is localized in the middle of the Hele-Shaw cell. This defect reduces locally the gap thickness of the cell and thus decreases its local permeability.

The obstacles are prepared by cutting and assembling pieces of $100-\mu \mathrm{m}$ self-adhesive transparent plastic film. For previous experimental series [25], glass plates of the cell were covered by a thin stretchable hydrophilic plastic film to ensure identical surface properties within our porous medium. Covering an obstacle by the film increased effectively the lateral size of the defect. Nevertheless, we checked that performing flow experiments without this cover film did not affect our results. Therefore, in the following experiments, we do not use this protocol for the sake of experimental simplicity.

\section{In situ foaming and forced flow}

A vertical chamber filled with a soapy solution obtained by mixing a commercial dish washing liquid (Dawn) with ultrapure water (1\% in volume) is connected to the cell via a $10-\mathrm{mm}$-wide and 120-mm-long hole drilled in the bottom plate of the cell.

We generate in situ a two-dimensional confined liquid foam, composed of a monolayer of quasi-mono-disperse bubbles, forced to invade the cell at a constant velocity, by bubbling filtered pressurized air in this tank through a needle at a constant rate imposed by a digital mass flow controller (Bronkhorst). In the present study, we report experimental results for constant flow rate $F_{\text {Air }}=40 \mathrm{ml} / \mathrm{min}$. The generated bubbles are then quasi-mono-disperse with a diameter larger than the cell gap, of around $3 \mathrm{~mm}$, mainly controlled by the inner diameter of the needle, $0.5 \mathrm{~mm}$.

The bubbles are forced to invade the cell at a constant longitudinal velocity $V_{0}=F_{\text {air }} / \mathrm{cs}=$ $3.25 \mathrm{~mm} / \mathrm{s}$, with the imposed air flow rate $F_{\text {Air }}$ and the cross section $c s$ of the cell, both quantities constant through the whole study. The gap $G$ is small enough for the bubbles to form a twodimensional confined foam, as a single monolayer of bubbles. Furthermore, by controlling the level of the surfactant solution in the tank, we can adjust the liquid fraction of the invading foam, without any significant impact on the imposed velocity $V_{0}$, since the liquid fraction remains much smaller than unity in all experiments.

Without any obstacle in the cell, the liquid foam displays a simple plug flow, with a constant velocity $V_{0}$ across the cell in the $y$ direction, the bubbles being simply translated without any deformation. This is associated to the main source of energy dissipation in our flow experiments: The friction at the contact between the bubbles and the glass walls in the confined dimension [26]. On one hand, this results in a rather constant pressure drop along the cell and negligible fluctuations 


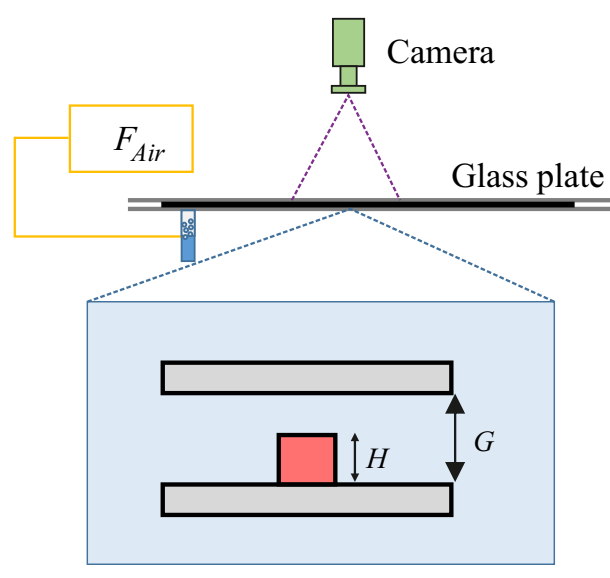

Side view

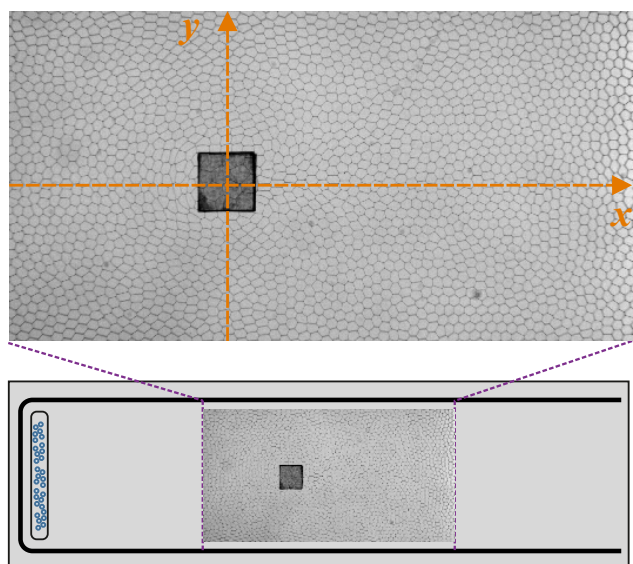

Top view

FIG. 1. Experimental setup: A model open fracture is made of two glass plates separated by a millimeter gap $G$, with a single permeable obstacle, i.e., a local constriction of lateral extent $a$ and height $H<G$, localized in the middle of the cell. A liquid foam is created in situ and forced to flow within this porous medium, by blowing air at a constant rate in a tank, filled with a soapy solution, connected to the bottom plate of the cell. The left panel shows a side view of the set-up, while the right one displays a top view together with a typical image recorded during a flow experiment (square obstacle with $a=20 \mathrm{~mm}, H / G=0.8, F_{\text {Air }}=$ $40 \mathrm{ml} / \mathrm{min}, \Phi=1.4 \times 10^{-2}$ ). Dashed orange lines represent the Cartesian coordinate system that is introduced with its origin in the center of the obstacle, with the $x$ axis along and $y$ axis perpendicular to the foam flow.

of the level of the surfactant solution in the tank. Furthermore, during our experiments, we have checked that this level did not evolve in the large tank, where the foam is generated. On the other hand, the lateral boundary condition in the $y$ direction sets by the toric nitrile joint does not slow down the bubbles in contact with this sealing border but simply acts as a no-flux boundary condition. As we will show below, this can lead to a blockage effect when the width of the obstacle becomes too large.

In the presence of a localized constriction, the velocity of the flowing foam is not uniform around such obstacle. Nevertheless, the permeable obstacle should modify neither the pressure drop along the cell, nor the far field velocity field as long as its radius is smaller than the cell length, as observed with similar setups with impermeable obstacles filling the gap of the cell [26]. In our experiments, we could indeed observe that, far from the constriction, where the perturbation has relaxed, we retrieve a constant longitudinal velocity, $V_{0}$, that we impose.

\section{B. Direct observation and image analysis}

We observe directly the steady-state flow of the two-dimensional liquid foam and specifically the motion and deformation of its elementary components, the bubbles, in the middle of the cell around the obstacle.

A digital camera (Basler) images an area of $200 \times 100 \mathrm{~mm}$, at a spatial resolution of $2048 \times$ 1088 pixels, and records 1000 frames during $250 \mathrm{~s}$, at a constant rate of $4 \mathrm{~Hz}$. A typical image recorded during a flow experiment of a dry liquid foam, invading the cell with a square obstacle $a=20 \mathrm{~mm}$, filling $80 \%$ of the cell gap $(H / G=0.8)$ is shown on the right panel of Fig. 1 . We also provide a typical recorded movie of such flow experiment in Supplemental Material [27].

Using standard image analysis tools proposed by ImageJ and Matlab softwares, we can obtain a network of skeletonized bubbles. A zoom around the obstacle of a typical recorded image and the 


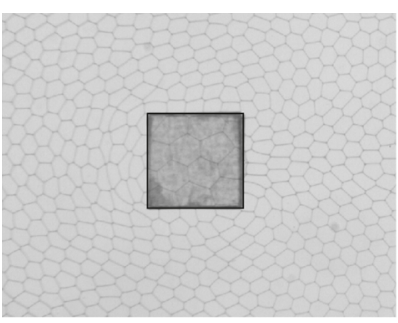

(a)

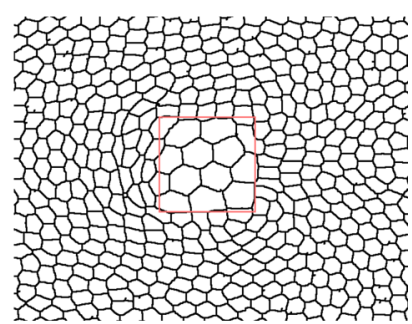

(b)

FIG. 2. Zoom on a region around a square constriction $(a=20 \mathrm{~mm})$ filling $80 \%$ of the cell gap for an image recorded during a flow experiment of a dry liquid foam: (a) A raw image and (b) a binary image, showing the results of our image analysis with a network of skeletonized bubbles.

results of edge detection are shown in Fig. 2. We can subsequently identify all individual bubbles of the flowing foam, for each recorded image, for the various experiments performed.

\section{Foam characterization}

The liquid foams we produce have a typical jammed disordered multiscale structure. Their elementary components, the bubbles, display mostly hexagonal shapes with six neighboring bubbles, as shown in inset of Fig. 3. Furthermore, we show in this figure that the distribution of the projected bubble area follows a Gaussian distribution, with an average size of $6.0 \mathrm{~mm}^{2}$ and a standard deviation of $0.6 \mathrm{~mm}^{2}$, with a negligible dependence with the liquid fraction.

Nevertheless, one can directly observe in Fig. 2 that the bubbles on the obstacle that are quasiincompressible have an increase of size, obviously related to the reduction of the gap cell thickness. More interestingly, one can also observe a symmetry breaking in the shape of the bubbles upstream vs downstream the obstacle; indeed, the bubbles approaching the constriction get compressed in the direction of the imposed mean flow, while the bubbles exiting the permeable defect are strongly elongated in the direction of the mean flow.

As mentioned, we can vary the foam liquid fraction by adjusting the level of the surfactant solution in the tank. The driest foam is obtained by letting the formulated foam drain in the tank over a height of $4 \mathrm{~cm}$, before entering the Hele-Shaw cell, while a wet foam is obtained by adjusting

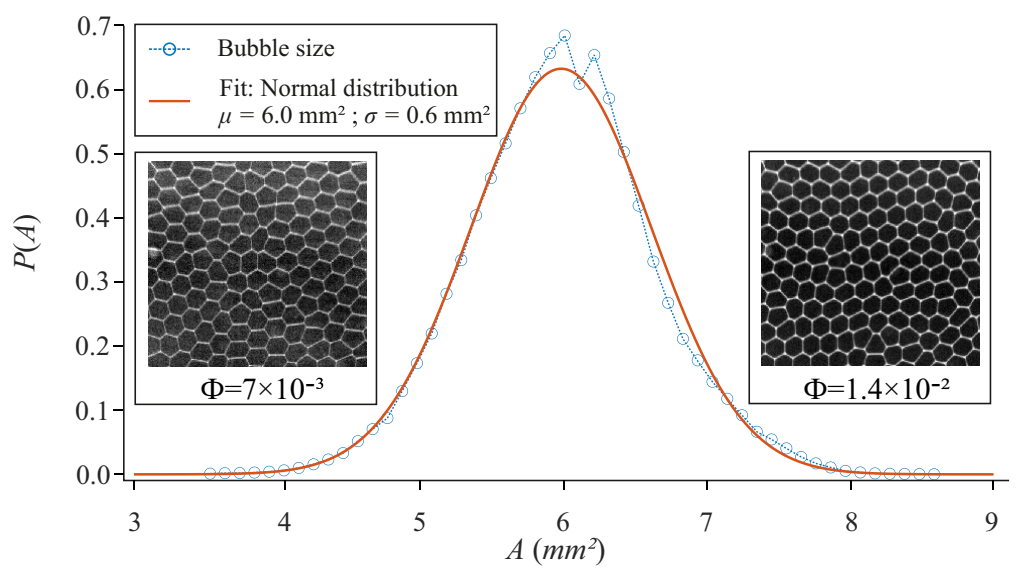

FIG. 3. Gaussian distribution of the projected area of bubbles for a dry foam invading a cell without any constriction. In inset, typical close-ups of a dry (left) and wet (right) foam show that those bubbles have mostly hexagonal shapes. 
the level of solution just below the cell inlet. We will study here flow experiments with foams of different liquid fractions, $\Phi=3 \times 10^{-3}, 7 \times 10^{-3}$, and $1.4 \times 10^{-2}$, referred as very dry, dry, and wet foam, respectively. The aforementioned values correspond to an effective liquid fraction [13], which definition is based on the average bubble area $A$ and on the minimal contact length $L_{c}$ between bubbles, before they undergo a topological rearrangement through neighbor swapping, a so-called T1 event [1]. With these notations the liquid fraction is given by $\Phi=0.242 L_{c}^{2} / A$ [13] and thus can be directly measured by extracting both $A$ and $L_{c}$ from the image sequences recorded for each experiment.

\section{Velocity measurement}

Tracking the foam bubbles, we can measure their velocity by computing their displacement between two recorded subsequent images. Nevertheless, instead of such tracking procedure, in order to extract the velocity fields of the flowing foam for various experiments we opted for an image correlation method. More specifically, we chose a time-resolved digital particle image velocimetry technique that treats the texture provided directly by the films of the bubbles themselves and their vertices. In practice, the Matlab toolbox, PIVlab [28] is used with the direct cross-correlation option, $64 \times 64$ pixels $^{2}$ interrogation area and 32-pixel step. This chosen area includes about three foam bubbles. Analyzing 1000 images recorded per experiment with a time lag of $\delta t=0.25 \mathrm{~s}$ between two images, we finally obtain a time-averaged steady-state velocity field $\bar{V}=\left(V_{x}, V_{y}\right)$. In the following, we will write $V_{x}=V$ for simplicity.

We have checked that analyzing solely the vertices of the skeletonized bubble network as tracers of the flow leads to similar velocity measurements. Such a result is particularly interesting to extract easily the velocity field of a three-dimensional liquid foam, flowing in a constriction or around an obstacle, such as the one captured recently using fast x-ray tomography [29], without the need of the very tedious and still unclear procedure of reconstruction of the three-dimensional bubbles shape [30].

\section{Permeability factor}

We define a permeability factor $Q$ that accounts for the foam flow rate through the constriction, in order to characterize with a global quantity the flow disturbance induced by such defect. Considering the longitudinal component of the velocity field $V$ of the flowing foam, along the vertical line (transverse direction to the mean flow) passing through the center of the obstacle ( $y$ axis, see the vertical dashed line in the right panel of Fig. 1), the constant flux of foam leads to:

$$
V_{0} \ell=\left(\int_{-\ell / 2}^{-a / 2}+\int_{a / 2}^{\ell / 2}\right) V d y+(1-H / G) \int_{-a / 2}^{a / 2} V d y,
$$

with $\ell$ the width of the Hele-Shaw cell and $V_{0}$, corresponding to the imposed longitudinal velocity. It is composed of the flow around and through the obstacle, respectively. The permeability factor $Q$ is then defined as the dimensionless flow rate passing through the obstacle:

$$
Q=\frac{(1-H / G) \int_{-a / 2}^{a / 2} V d y}{V_{0} a}=\frac{\ell}{a}-\frac{\left(\int_{-\ell / 2}^{-a / 2}+\int_{a / 2}^{\ell / 2}\right) V d y}{V_{0} a} .
$$

The factor $Q$ is equal to 1 if the obstacle is fully permeable $(H=0$, no obstacle) and 0 if impermeable ( $H=G$, obstacle filling completely the cell gap). This last equation shows that this permeability factor $Q$ can be obtained from the velocity field of the liquid foam measured either inside or outside the obstacle.

\section{Bubble deformation measurements}

By studying the shape of each labeled bubbles of the flowing foam, we can quantify its deformation. Indeed, approximating each bubble as an ellipse, we compute their eccentricity $\epsilon$ to 


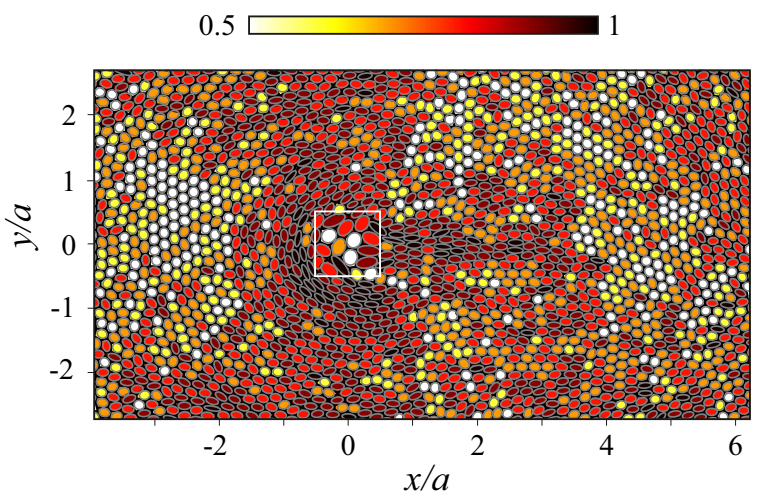

FIG. 4. Example of an instantaneous bubble deformation map, obtained for a dry foam invading an HeleShaw cell with a square obstacle of lateral size $a=20 \mathrm{~mm}$, filling $60 \%$ of the cell gap, where we display both the skeletonized bubble network and the ellipses fitting each labeled bubbles and their eccentricity is given by the color bar.

obtain a measure of the bubble deformation. The eccentricity is defined as the ratio of the distance between the foci of the ellipse and its major axis length, $\epsilon=\sqrt{1-b^{2} / a^{2}}$, where $a$ and $b$ are the length of the major and minor axes, respectively. By definition, the eccentricity takes a value between two extreme limit cases, 0 (for a circle) and 1 (for a line segment). We then attribute to each pixel of each labeled bubbles of an image recorded at a time $t$, the corresponding value of the measured eccentricity. Thus, we obtain a spatial map of the bubble deformation $D$ at time $t$. An example of such map is shown in Fig. 4.

Finally, we average the computed spatial maps of bubbles' eccentricity to obtain a time-averaged steady-state bubble deformation field $\bar{D}$ of the foam flowing through and around the constriction.

Contrary to the bubble velocity measurements based on the PIV technique, such analysis of the bubble deformation is very sensitive to any defect in the edge detection and the bubbles labeling procedure; it indeed requires an accurate identification of a closed boundary for each bubbles of the flowing foam. Despite a very good quality and contrast of the recorded images, a tedious manual check was necessary to detect few defects and close correctly the skeletonized bubbles. Therefore, in the present study, we analyzed a subsample of 200 images over the thousand recorded, for each experiment, separated by $\delta t=0.5 \mathrm{~s}$.

\section{STEADY-STATE VELOCITY AND BUBBLE DEFORMATION FIELDS}

\section{A. Geometrical effects}

First, we investigate the impact of the geometry of the obstacle - and, in particular, the obstacle height —on the bubble deformation and velocity fields of the foam.

\section{Obstacle height}

a. Velocity fields. We show in Fig. 5 the average steady-state velocity fields $\bar{V}$ of a dry foam invading the cell with a square obstacle of lateral size $a=20 \mathrm{~mm}$ and increasing height $H$, from top (no obstacle, $H / G=0$ ) to bottom (obstacle filling the gap of the cell, $H / G=1$ ), with the following ratio of the obstacle height over the cell gap, $H / G=0,0.3,0.6,0.8,1$.

Whatever the value of $H / G$, far from the obstacle, we observe that the foam velocity is constant, corresponding to the imposed longitudinal velocity $V_{0}$. Nevertheless, approaching such permeable obstacle, the amplitude of the velocity field decreases, while it increases when leaving it. 

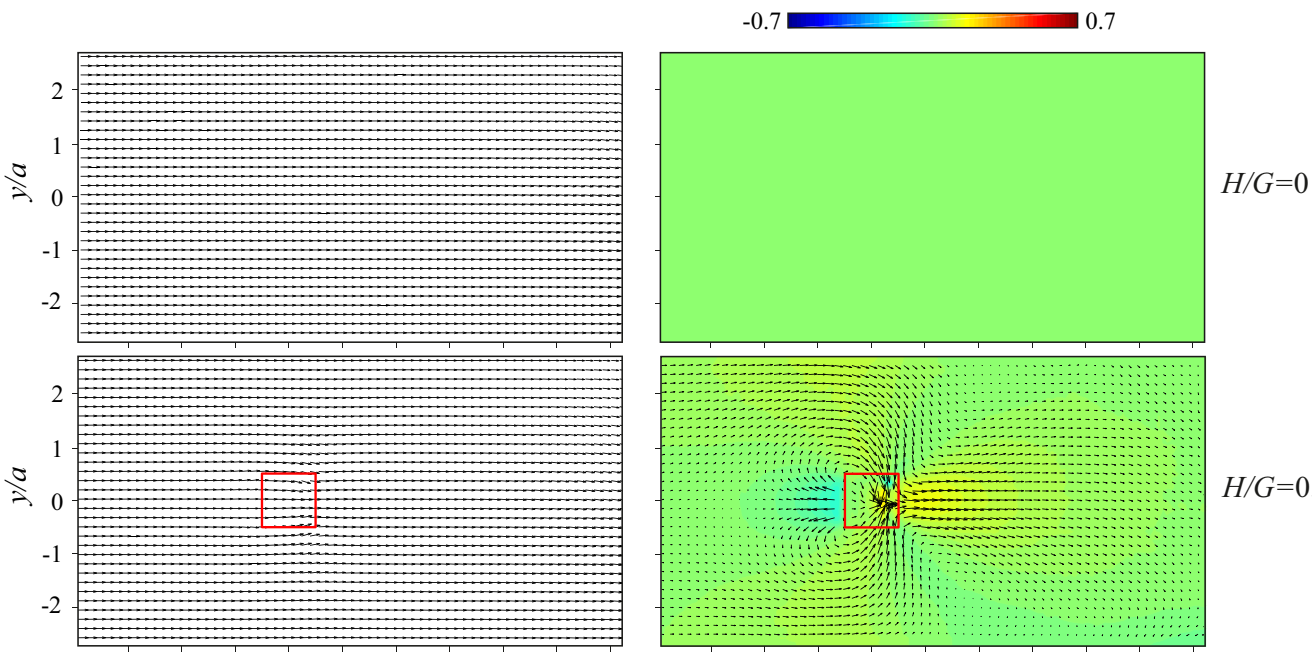

$H / G=0.3$
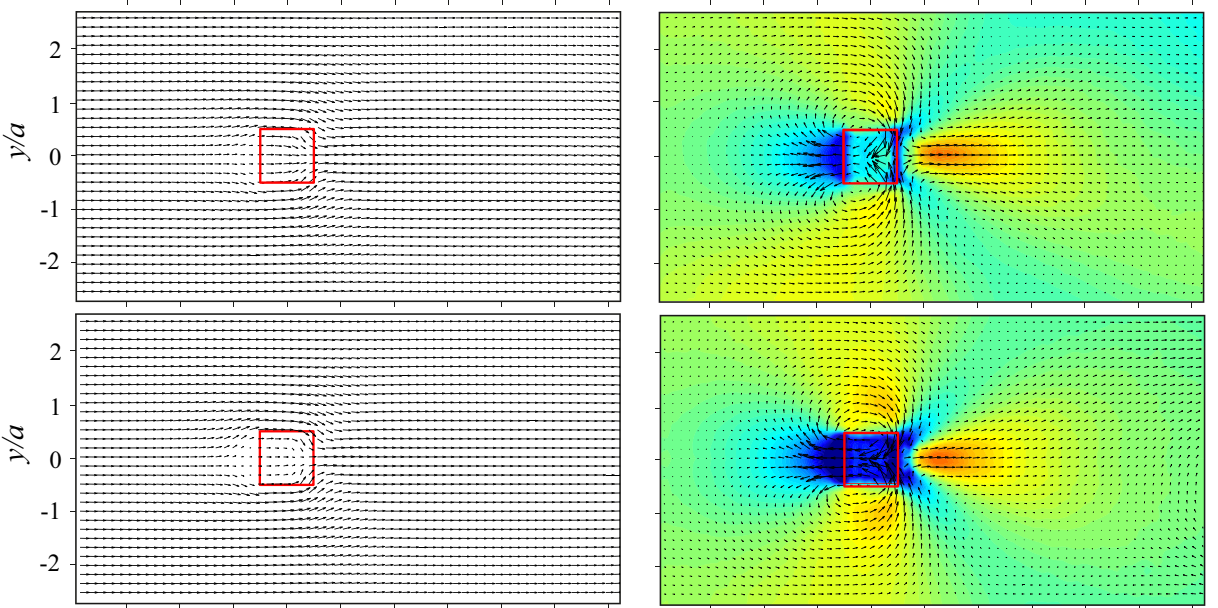

$H / G=0.6$
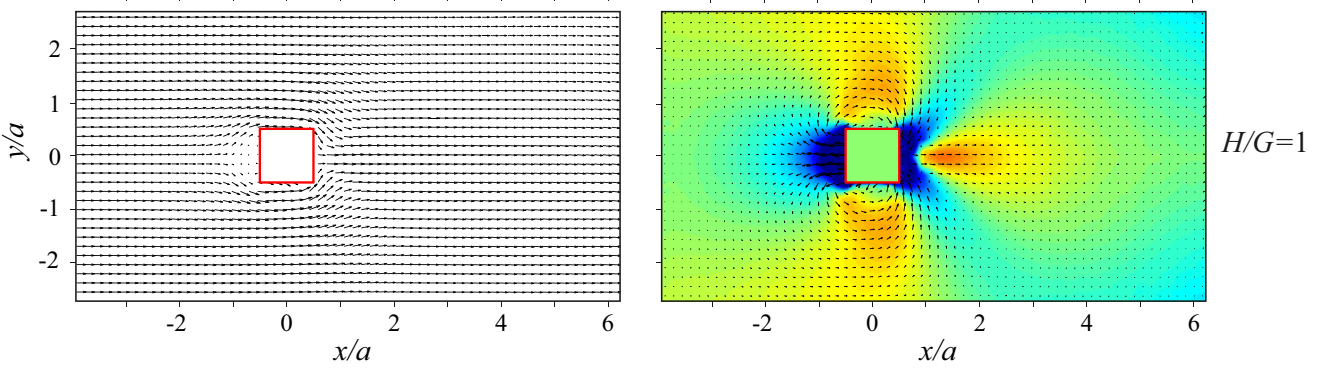

FIG. 5. Average velocity fields $\bar{V}$ in the frame of the laboratory (left column) and in the frame of the flowing foam (right column), for a dry foam invading the cell with a square obstacle of lateral size $a=20 \mathrm{~mm}$ and increasing height $H$, from top (no obstacle, $H / G=0$ ) to bottom (obstacle filling the gap of the cell, $H / G=1$ ), with the following ratio of the obstacle height over the cell gap, $H / G=0,0.3,0.6,0.8,1$. The colored maps on the right panels give also the amplitude of the normalized averaged longitudinal velocity $\left(V-V_{0}\right) / V_{0}$, where $V_{0}$ is the imposed longitudinal velocity.

The panels on the right of Fig. 5 display the velocity fields in the frame of the flowing foam, where we have subtracted this far field velocity $V_{0}$. Colored maps are superimposed and give the amplitude of the normalized averaged longitudinal velocity $\left(V-V_{0}\right) / V_{0}$. As soon as an obstacle is present 


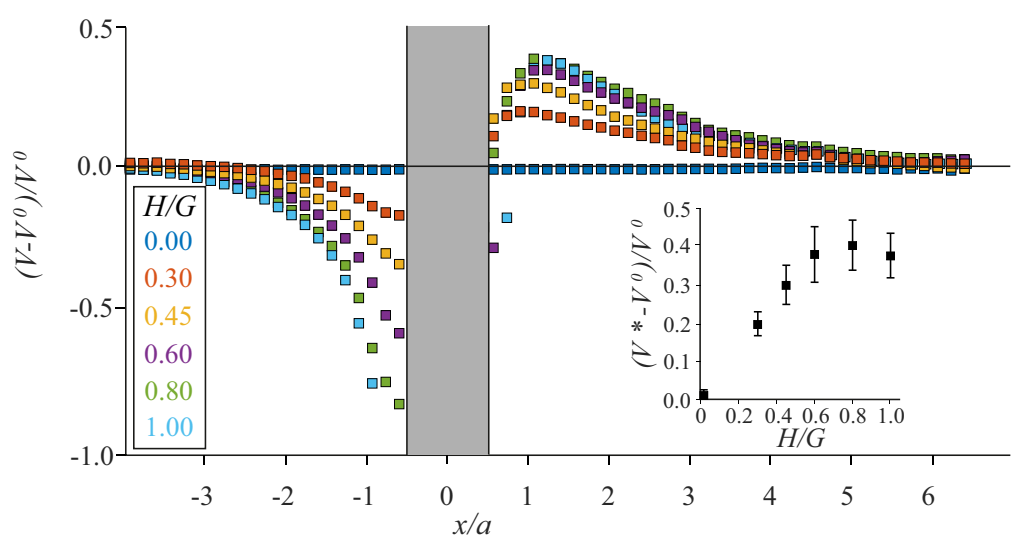

FIG. 6. Profiles of normalized longitudinal velocity $\left(V-V_{0}\right) / V_{0}$ measured along the mean direction of the flow (horizontal line $y / a=0$, see Fig. 1) through the center of the cell, with a square obstacle $(a=20 \mathrm{~mm})$ of different height $H$. Inset: Maximum value of those normalized velocity profiles $\left(V^{*}-V_{0}\right) / V_{0}$ as a function of the obstacle height $H / G$.

within the cell, even reducing slightly its gap, we can clearly notice the disturbance of the velocity field, in particular in the frame of the flowing foam: a clear recirculation is then revealed, with a strong fore-aft asymmetry of the flow, evidenced by a zone downstream the obstacle, where the foam velocity can be much larger than the imposed longitudinal velocity $V_{0}$. Such effect is reminiscent of the "negative wake" observed in the flow pattern of rising air bubbles in viscoelastic fluids [31]. More relevantly, our observations appear analogous to the results obtained for a two-dimensional foam flowing around an impermeable obstacle, characterized by Dollet et al. [12,13] using a liquid pool geometry. The observed asymmetry of the velocity field and its overshoot downstream the obstacle was attributed to the viscoelastoplastic nature of the foam [16], which cannot be modeled as a simple viscoplastic fluid [32].

Interestingly, in our flow experiments with a permeable obstacle, we can observe that both the amplitude of the overshoot and the region where it develops evolve nontrivially with the gap of the constriction. In particular, they tend to saturate and eventually decrease for very strong confinement, when the height of the constriction over the gap of the cell $H / G>0.7$. This is clearly shown and quantified in Fig. 6, where we display the evolution of the normalized longitudinal velocity profiles along the direction of the mean flow $\left(V-V_{0}\right) / V_{0}$, measured in the central part of the cell $(y=0)$ as a function of the height of the obstacle $H / G$. Specifically, in the inset of Fig. 6, we display the nonmonotonic behavior of the maximum value of those normalized longitudinal velocity profiles as a function of the constriction height $H / G$.

b. Bubble deformation fields. We now investigate the time-averaged steady-state bubble deformation fields for the very same series of flow experiments. These fields are shown in the right panels of Fig. 7, where the color code gives the amplitude of the time-averaged eccentricity of the bubbles, approximated by ellipses. On those colored maps, the superimposed ellipses correspond to the labeled bubbles, detected for a given image recorded during a flow experiment, actually shown in the left panels. The figures on the top correspond to an experiment, where the liquid foam flows in a cell without any constriction. The corresponding bubble deformation field appears rather homogeneous with an average value of the eccentricity around $0.60 \pm 0.04$, (though one can observe some deviations with notably larger values along the cell boundaries, probably related to small inhomogeneities, when tightening manually the gap cell). Our analysis does not account for the arrangement structure with the neighboring bubbles, considering each labeled bubbles independently. This somehow could explain the rather high value of average eccentricity, characterizing the typical bubbles' deformation of a dry foam forced to invade our homogenous Hele-Shaw cell. 

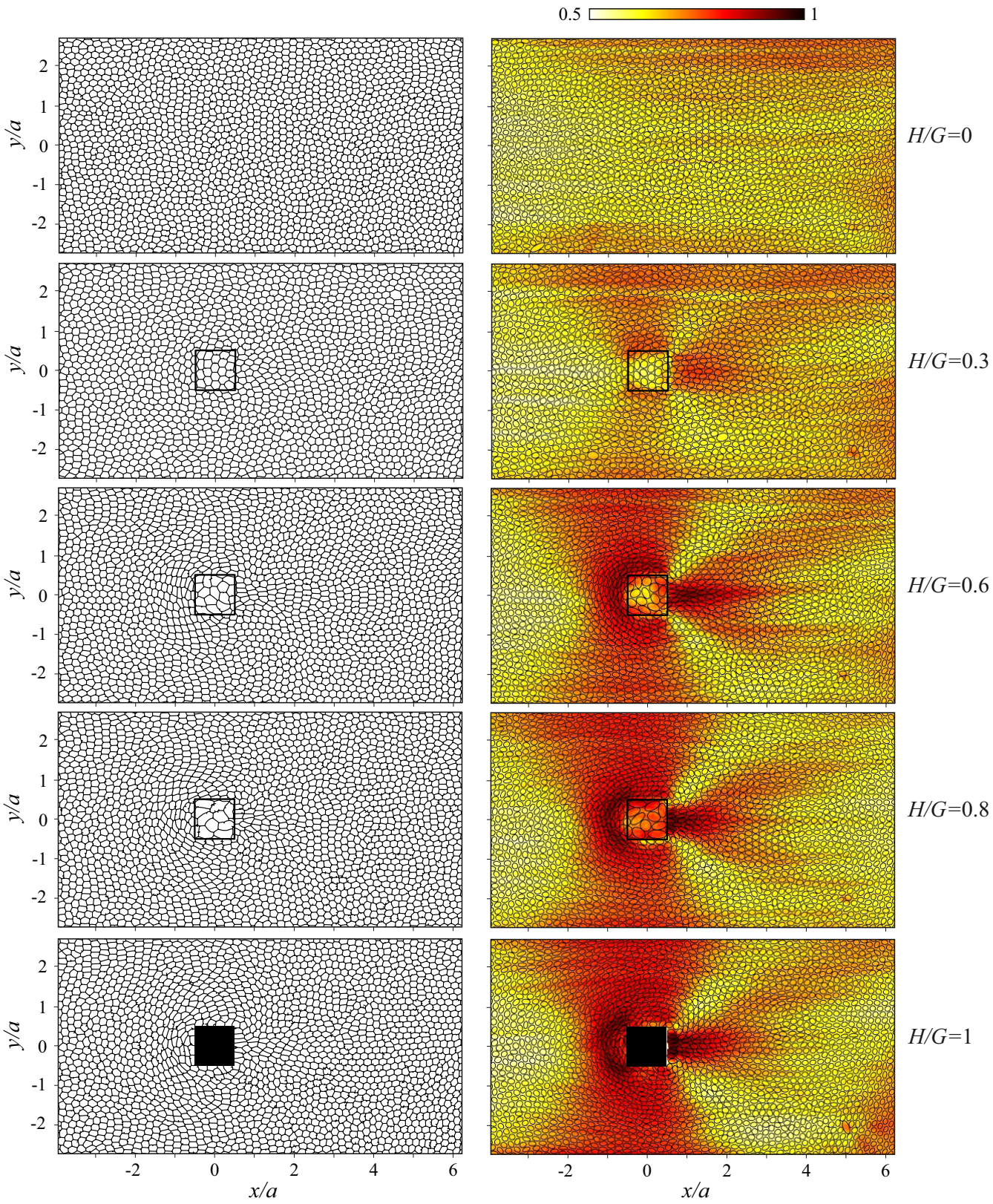

FIG. 7. Average deformation fields for a dry foam invading the cell with a square obstacle of lateral size $a=20 \mathrm{~mm}$ and increasing height $H$, from top (no obstacle, $H / G=0$ ) to bottom (obstacle filling the gap of the cell, $H / G=1$ ), with the following ratio of the obstacle height over the cell gap, $H / G=0,0.3,0.6,0.8,1$. The color code gives the amplitude of the time-averaged eccentricity of the bubbles (considered as ellipses). On the right panels we superimpose to the average deformation fields the ellipses corresponding to each labeled bubbles detected for a given image recorded during a flow experiment and shown on the left panels.

As for the velocity fields shown just before, the bubble deformation field of the foam is clearly impacted by the presence of even a mild local reduction of the cell gap. We again clearly observe a complex structure for those fields, with in particular a fore-aft asymmetry, which reflects the symmetry breaking in the shape of the bubbles upstream vs downstream the obstacle, that we already 


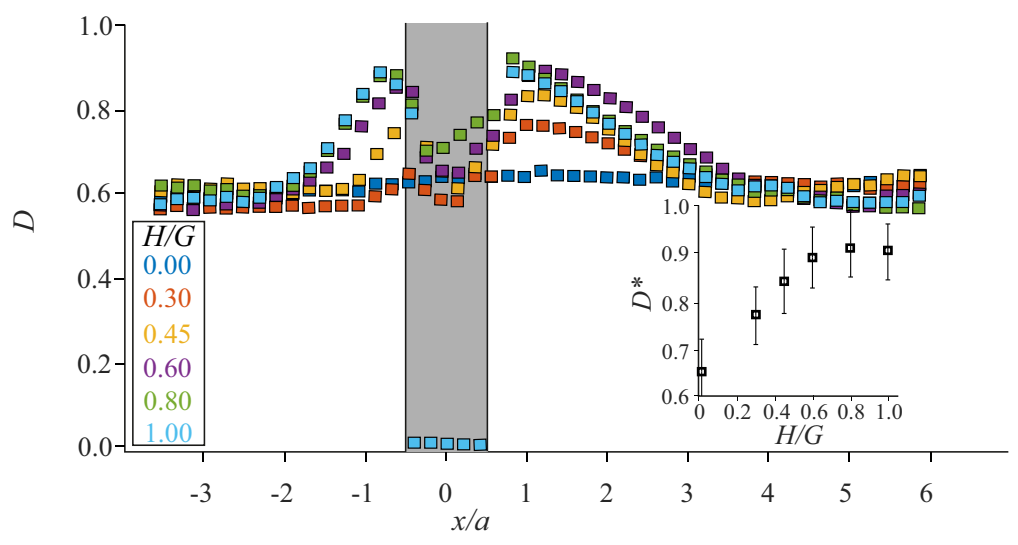

FIG. 8. Profiles of the bubble deformation, measured along the mean direction of the flow (horizontal line $y / a=0$, see Fig. 1) through the center of the cell, with a square obstacle $(a=20 \mathrm{~mm})$ of different height $H$. Inset: Maximum value of the deformation measured along those deformation profiles $D^{*}$ as a function of the obstacle height $H / G$.

noticed in Fig. 2: The bubbles approaching the constriction are compressed in the direction of the imposed mean flow, while the bubbles exiting the permeable defect are strongly elongated in this direction of the mean flow. Furthermore, we can clearly distinguish a semicircular region upstream the obstacle, where the bubbles are strongly stretched along a direction indicating that those bubbles will flow around the constriction.

Indeed, those deformation maps are clearly correlated to the velocity fields shown in Fig. 5, especially considering the region downstream the obstacles, where the foam velocity can be much larger than the imposed longitudinal velocity $V_{0}$, concomitantly to a strong elongation of the bubbles along this longitudinal direction of the imposed flow $\bar{V}_{0}$.

Interestingly, we can also notice that those bubble deformation maps look very similar for constrictions reducing locally the gap of 60,80 , and $100 \%$. This observation is clearly confirmed when we represent, in Fig. 8, the profiles of the bubble deformation, measured along the mean direction of the flow (horizontal line $y / a=0$ ) through the center of the cell, with a square obstacle ( $a=20 \mathrm{~mm}$ ) of systematically increasing height $H$. As for the velocity fields, the nonlocal impact and disturbance of the constriction-like defect is clearly evidenced, especially downstream the obstacle, where the bubbles recover a relaxed state (corresponding for this dry foam, here, to a value of an average eccentricity around 0.6) at a typical distance of around four times the obstacle size.

Moreover, the maximum value of the bubble deformation (given by the averaged eccentricity of the bubbles) along those longitudinal profiles saturates when the constriction reduces the gap of $60 \%$, as shown in insert, similarly to the evolution of the maximum value of the velocity overshoot observed in Fig. 6.

c. Permeability of the inhomogeneous cell. We show in Fig. 9 a series of profiles of the longitudinal velocity, centered on the obstacle $(x=0)$, in the direction $y$ transverse to the mean flow, for the same series of experiments (a dry foam invades the cell with a square obstacle of lateral size $a=20 \mathrm{~mm}$ and various height $H$ ). Those velocity profiles are normalized as $\left(V-V_{0}\right) / V_{0}$.

As expected, those transverse velocity profiles have a symmetric shape, with the velocity of the foam flowing through the permeable obstacle decreasing systematically with its height, while increasing sideways up to a distance of around three times the obstacle lateral extent. Those measurements allow us to estimate a permeability factor $Q$ of our medium, as a function of the ratio of the obstacle height over the cell gap $H / G$.

This permeability factor $Q$ shown in the inset of Fig. 9 is obtained by computing the volume flow rate of the liquid foam through and outside the obstacle as explained in Sec. II B 3. The reported 


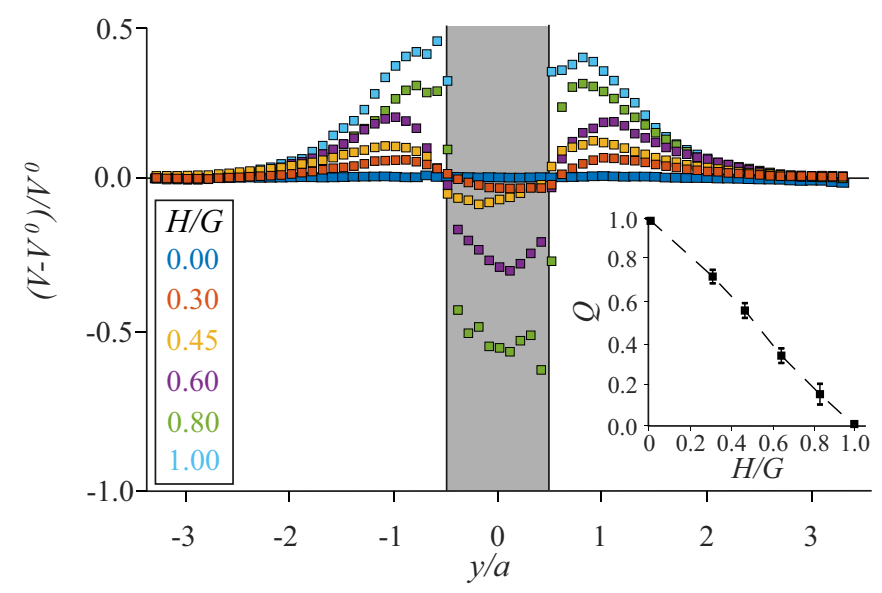

FIG. 9. Profiles of the normalized longitudinal velocity $\left(V-V_{0}\right) / V_{0}$, centered on the obstacle $(x=0)$, in the direction $y$ transverse to the mean flow, as a function of the height of the constriction $H / G$. The inset shows the permeability factor $Q$ for those experiments, where a dry foam invades the cell with a square obstacle of lateral size $a=20 \mathrm{~mm}$.

values correspond to the mean between the two different ways of measurement, while the error bars reflect the standard deviation. For these flow experiments, we observe a systematic quasilinear decrease of the permeability factor $Q$ as the gap of the cell is locally reduced.

\section{Obstacle shape and size}

To further complete our study of the impact of the geometry of the obstacle on the foam flow, we report, in the following, some experimental results for obstacle of different shapes as well as different lateral extents.

Figure 10 displays the average longitudinal velocity fields of a flowing foam for two different obstacle shapes - circular versus square-with all the other parameters of the experiments being identical (driving velocity, liquid fraction, height, and diameter or lateral extent of the obstacle), displayed in the frame of the flowing foam. At first order, the measured velocity fields are similar. A small difference can be noticed though in the recirculation observed sideways the obstacle, as well as for the overshoot, which has a larger extension in the mean direction of the flow for the square obstacle. In this case, we observe a "channeling" flow within the obstacle, with bubbles following the obstacle straight boundaries. The profiles of the longitudinal velocity in the center of the cell, along the direction of the flow shown for those two different obstacles (Fig. 10, bottom panel) confirm that the amplitude of the disturbance is slightly larger for the square obstacle-probably simply related to the fact that such defect has an effective size larger than for a circular one of an equivalent diameter.

We also report how the averaged steady-state velocity field of the foam evolves with the lateral extent of the constriction. Figure 11 shows the normalized longitudinal velocity field for a square obstacle of lateral extent $a=10 \mathrm{~mm}$ (top panel) and $a=30 \mathrm{~mm}$ (middle panel). The case $a=$ $20 \mathrm{~mm}$ is already given in the middle panel of Fig. 10. The size of the obstacle has obviously a strong impact on the foam velocity field on both its structure and amplitude. Indeed, due to the moderate values of the ratio obstacle diameter over the cell width, a consequent blockage effect can be observed, with a foam velocity close to the cell boundary larger than $V_{0}$. The larger the obstacle diameter, the stronger the effect.

Moreover, we show in the bottom panel of Fig. 11, profiles of normalized longitudinal velocity $\left(V-V_{0}\right) / V_{0}$, along the mean direction of the flow, rescaled by the lateral extent of the constriction $x / a$. These rescaled profiles for three different experiments with $a=10,20$, and $30 \mathrm{~mm}$ are very 

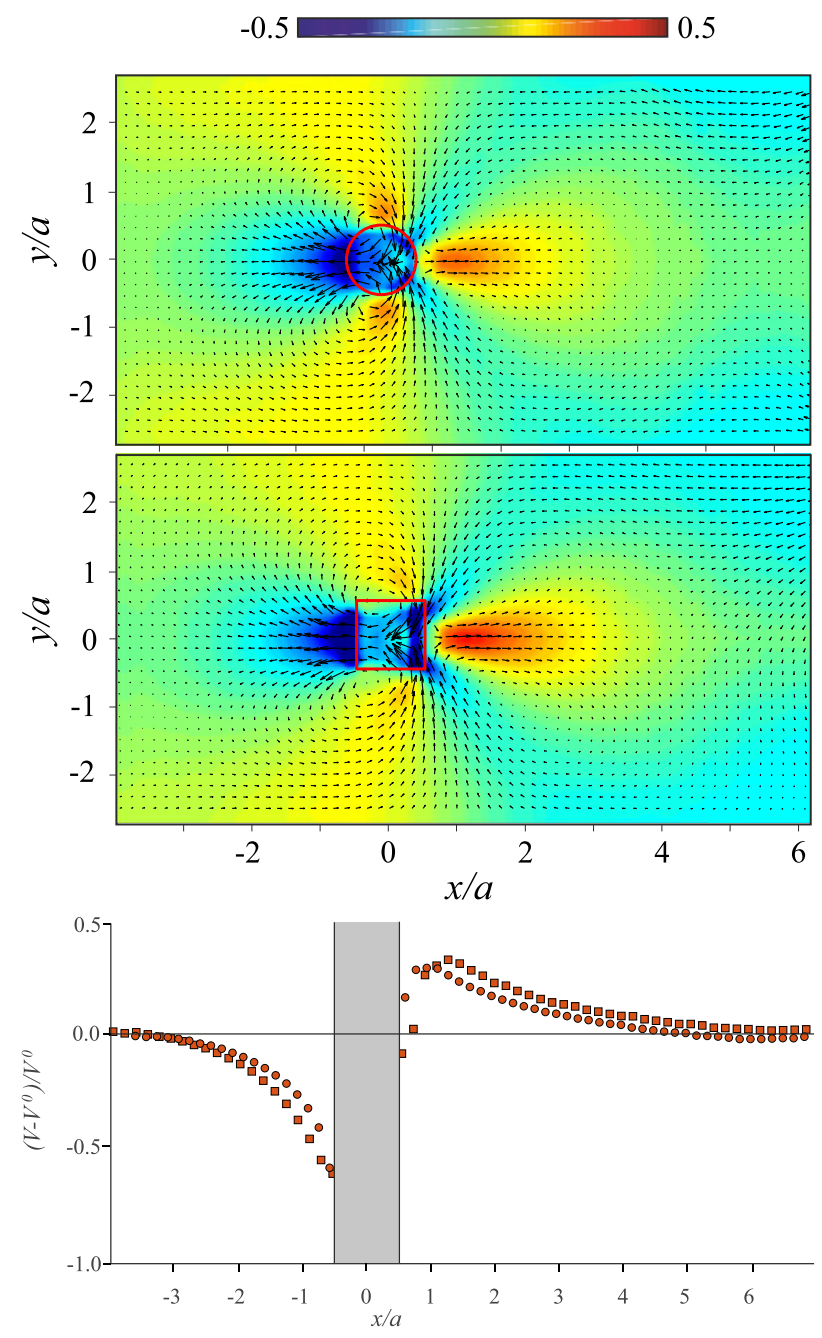

(a)

FIG. 10. (a) Average velocity fields $\bar{V}$ (in the frame of the flowing foam) of a dry foam invading the cell with a circular obstacle of diameter $a=20 \mathrm{~mm}$ and a square obstacle of lateral size $a=20 \mathrm{~mm}$; in both cases, the obstacle height to gap ratio is $H / G=0.6$; the colored maps give the amplitude of the normalized averaged longitudinal velocity $\left(V-V_{0}\right) / V_{0}$. (b) Corresponding normalized longitudinal velocity profiles (in the center of the cell $y=0)$. The symbols shape corresponds to the type of obstacles.

close to each other, revealing that, at first order, the flow disturbance is proportional to the size of the obstacle. Nevertheless, even after renormalizing those velocity profiles, we still can observe in the inset of the bottom panel of Fig. 11 a systematic increase of the maximum amplitude of the overshoot with the lateral extent of the constriction.

We can furthermore notice the observation of finite-size effects. Indeed, on one hand, the decreasing velocity trend at the leading edge of the smallest obstacle appears slightly different from one observed with the larger obstacles. Such an observation could be related to a discrete effect, since for experiments with small obstacle with $a=10 \mathrm{~mm}$, its lateral extent becomes comparable to the bubble size, outside the constriction. Inside the constriction, the projected area increases and very few (at most three) bubbles are found within the confining space. On the other hand, one may notice that the wake at the trailing edge of the largest obstacle with $a=30 \mathrm{~mm}$ relaxes toward the 


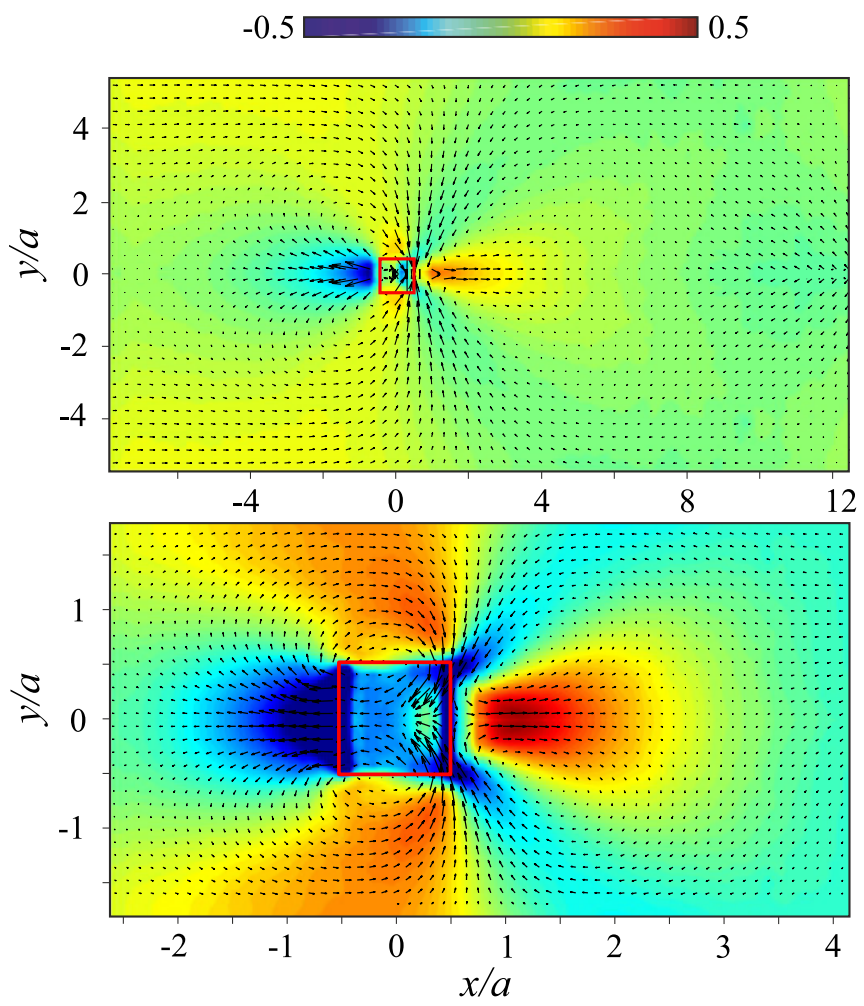

(a)

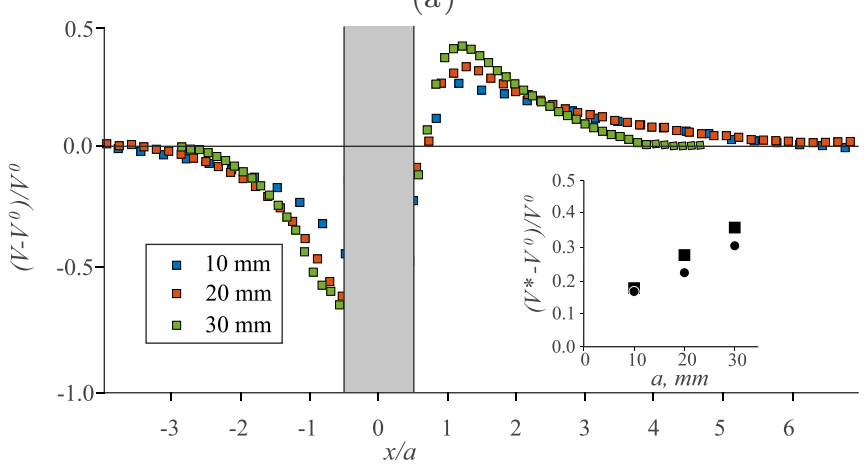

(b)

FIG. 11. (a) Average velocity fields $\bar{V}$ for a dry foam invading the Hele-Shaw cell with a square obstacle of lateral sizes $a=10$ (top panel) and $30 \mathrm{~mm}$ (bottom panel) and height $H / G=0.6$, displayed in the frame of the flowing foam. The colored maps give the amplitude of the normalized averaged longitudinal velocity $\left(V-V_{0}\right) / V_{0}$. (b) The bottom panel shows the corresponding normalized longitudinal velocity profiles along the mean direction of the flow in the center of the cell $(y=0)$; for different lateral sizes $a$ of the obstacle. The inset shows the maximum value of the velocity overshoot as a function of the lateral size of the obstacle. The symbols shape corresponds to the type of obstacles.

far-field imposed flow on a shorter rescaled distance. One possible explanation is that the flow is confined in the lateral direction, as observed in Fig. 11, due to the finite width of the cell. This effect reduces the extent of the perturbation laterally, but could also reduce its extent in the longitudinal direction. 


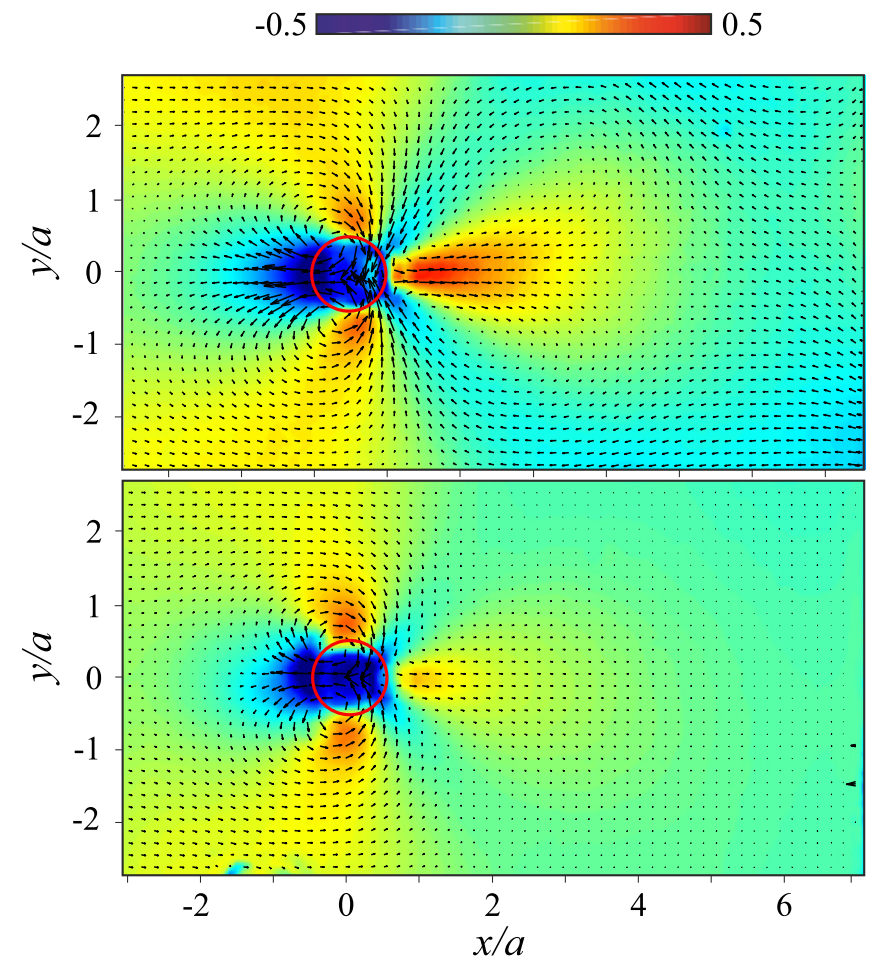

(a)

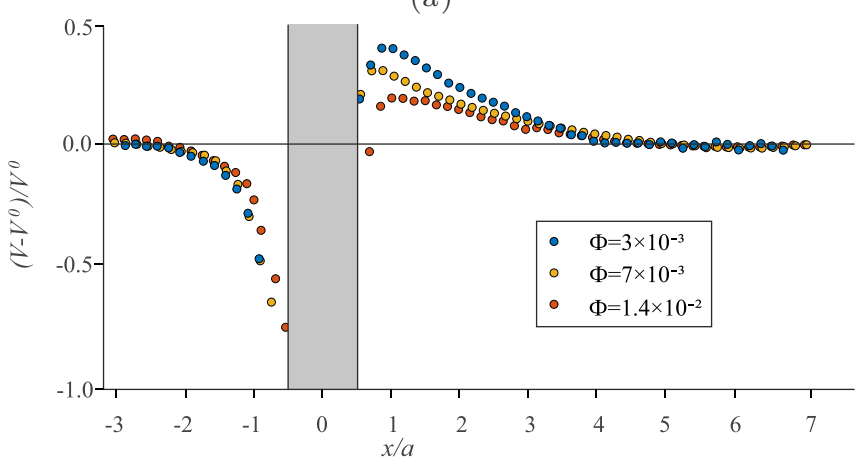

(b)

FIG. 12. (a) Average velocity fields $\bar{V}$ for a very dry and a wet foam with a liquid fraction of $\Phi=$ $3 \times 10^{-3}$ and $1.4 \times 10^{-2}$, top and middle panels, respectively, invading the cell with a circular obstacle of diameter $a=20 \mathrm{~mm}$ and height $H / G=0.6$, displayed in the frame of the flowing foam; the colored maps give the amplitude of the normalized averaged longitudinal velocity $\left(V-V_{0}\right) / V_{0}$. (b) This panel shows the corresponding normalized longitudinal velocity profiles along the mean direction of the flow in the center of the cell $(y=0)$;

\section{B. Liquid fraction effect}

Finally, we also investigate how the liquid fraction of the foam affects its flow, e.g., the motion and deformation of the bubbles, through and around the constriction.

Figure 12 shows average steady-state velocity fields for a very dry $\Phi=3 \times 10^{-3}$ and a wet $1.4 \times 10^{-2}$ liquid foam, invading the Hele-Shaw cell with a circular obstacle of diameter $a=20 \mathrm{~mm}$ and height $H / G=0.6$. Notice that the case of a dry foam is already shown in the top panel of 
Fig. 10. The bottom panel of Fig. 12 displays also the profiles of the normalized longitudinal velocity in the mean direction of the flow in the center of the cell $(y=0)$. Again, we can observe the disturbance of the foam flow due to the localized constriction (in this case, the gap is locally reduced of $60 \%$ ), with a strong asymmetry of the velocity field upstream vs downstream the obstacle. Nevertheless, our measurements show that when increasing the foam liquid fraction, this fore-aft asymmetry of the velocity field and more globally, the amplitude of the flow disturbance created by the obstacle is reduced. Specifically, we notice that, while the spatial extent of the overshoot downstream the constriction does not seem to be affected by the foam liquid fraction, its amplitude decreases strongly for a wetter foam.

We go further in our analysis by showing for the same series of flow experiments, the average bubble deformation fields $\bar{D}$ in Fig. 13. The bottom panel of this figure displays also the profiles of the average bubble deformation in the center of the cell $(y=0)$, along the mean direction of the flow. We can observe anew the complex structure of those bubble deformation maps correlated to the average steady-state velocity fields of the liquid foam-a multipolar structure, with a fore-aft asymmetry. The important result here is the fact that when increasing the liquid fraction of the foam, the disturbance of the bubble deformation field due to the constriction is reduced, as for the velocity field. The amplitude of the bubble deformation increases with a drier foam, while the extent of the region where the bubbles are strongly elongated (either upstream or downstream the obstacle) does not seem to evolve with the liquid fraction of the foam. We can further notice that the amplitude of the bubble deformation in the relaxed state far from the obstacle decreases with the liquid fraction - the bubbles getting closer to a circular shape, their average value of eccentricity decrease.

Both the elastic shear modulus and the yield stress of liquid foams decrease with their liquid fraction [3,33]. Therefore, as we will discuss in the following conclusive section, our various experimental observations and measurements point out the role of the elastic properties of liquid foams, which appear responsible for such symmetry breaking of the flow.

Our experiments show a clear correlation between the deformation of the bubbles and the liquid foam velocity.

Specifically, the top and middle panels of Fig. 14 demonstrate that the maximum amplitude of the velocity overshoot and the maximum value of the bubble deformation (both measured downstream the obstacle) display the same evolution with the gap contrast between the cell and the localized constriction $H / G$, as well as with the foam liquid fraction $\Phi$, for various experiments-performed with different obstacle shapes and liquid fractions of the foam. Indeed, both quantities increase and then saturate for strong confinement, when $H / G$ becomes larger than $60 \%$ and, moreover, systematically decrease for wetter foams. We furthermore confirm that the shape of the obstacle has a rather minor effect on the foam flow. Finally, the bottom panel of Fig. 14 shows that the amplitude of the velocity overshoot $V^{*}$ is proportional to the maximum bubble deformation $D^{*}$; our experimental data are indeed very well fitted by the following expression, $V^{*} / V_{0}=1.55 D^{*}$.

At the light of our various experimental results, we can propose the following mechanism at the origin of the rheological behavior observed: The localized constriction-like defect disturbs the foam flow by notably inducing a strong elongation of the bubbles, which increases with the amplitude of the disturbance, mainly controlled by the height of the obstacle. The subsequent elastic stressstored upstream and released downstream the constriction-leads to the foam flow asymmetry and in particular the velocity overshoot. One should note that our bubble deformation analysis (purely scalar) does not lead to a straightforward estimation of an elastic stress. A tensorial approach such as the one developed in $[14,15,34]$ would have been indeed necessary to measure an elastic internal strain, based, for instance, on the calculation of a texture tensor, from the links between neighboring bubbles. However, the simplicity of implementation of our analysis allowed us to obtain easily a 


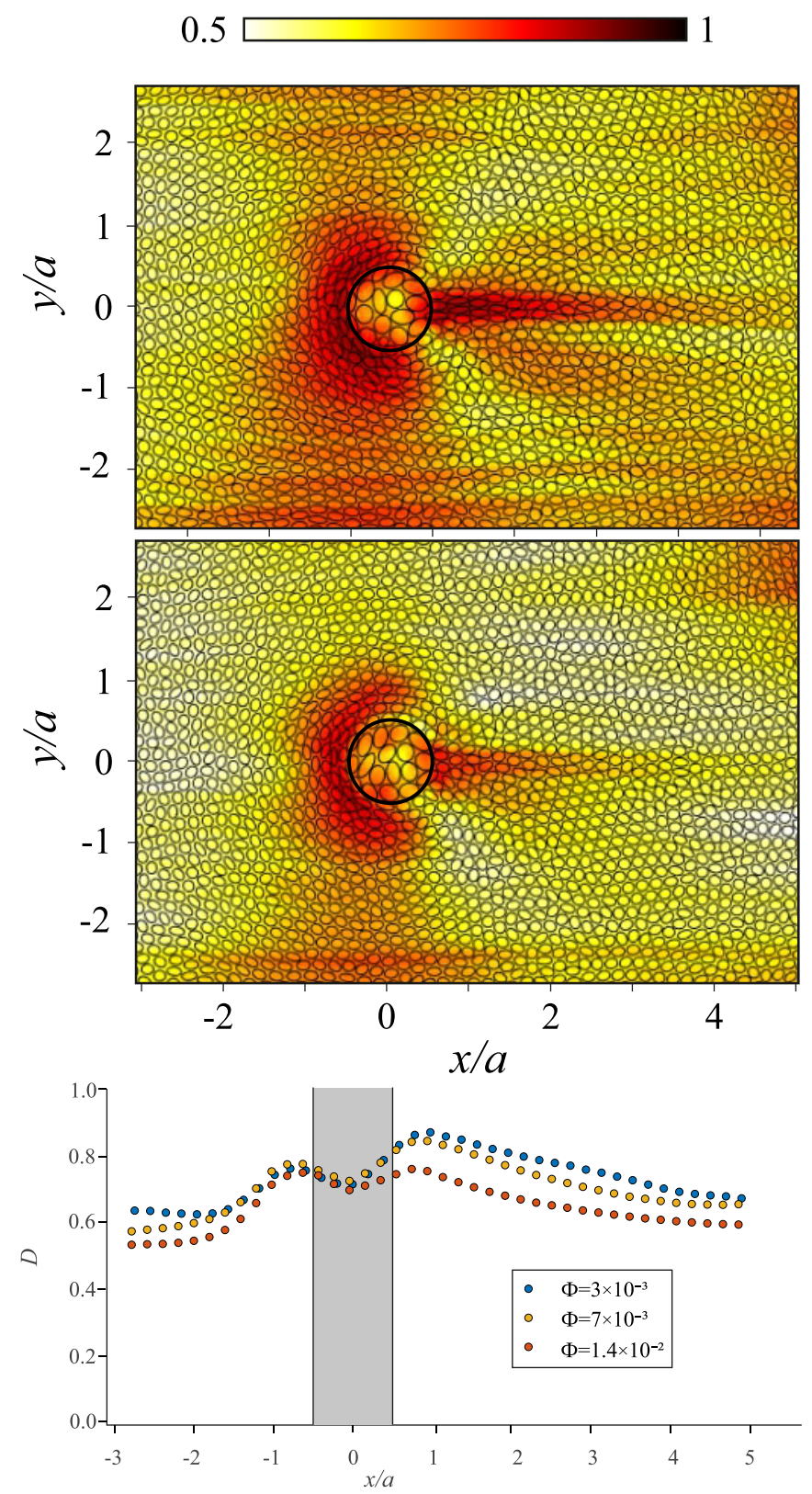

FIG. 13. Average bubble deformation fields $\bar{D}$ for a dry (top panel) and a wet (middle panel) foam with a liquid fraction of $\Phi=7 \times 10^{-3}$ and $1.4 \times 10^{-2}$, respectively, invading the cell with a circular obstacle of diameter $a=20 \mathrm{~mm}$ and height $H / G=0.6$; the colored maps give the amplitude of the averaged eccentricity. The bottom panel shows the corresponding average bubble deformation profiles along the mean direction of the flow in the center of the cell. Large bubbles deformations measured on some boundaries are just due to small defects in the gap cell, related to its manual tightening.

qualitative characterization of the deformation of the bubbles in order to evidence the role of the localized constriction.

Moreover, we can also explain the plateau in the maximal deformation of the bubbles and foam velocity, observed for strong confinement, by considering the plastic behavior of the liquid foam. 

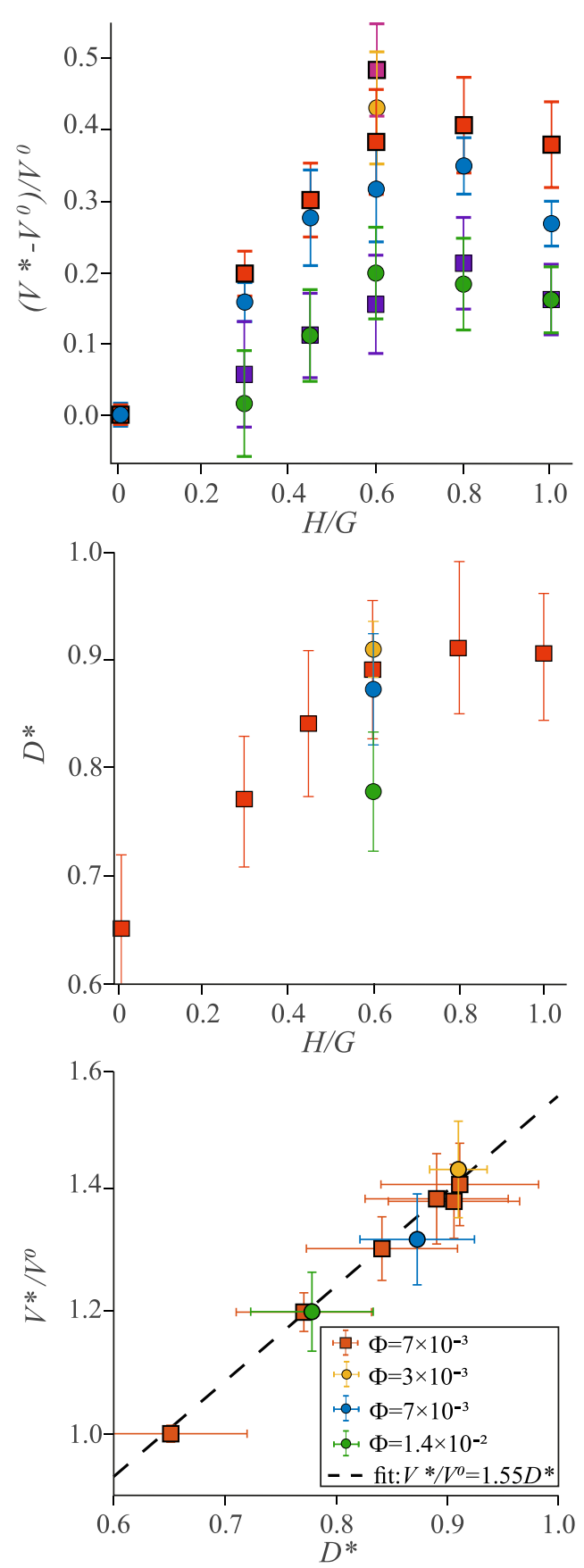

FIG. 14. Maximum value of the normalized velocity overshoot $\left(V^{*}-V_{0}\right) / V_{0}$ (top panel) and bubble deformation $D^{*}$ (middle panel) as a function of the obstacle height $H / G$ for square and circular objects with $a=20 \mathrm{~mm}$ for $\Phi=3 \times 10^{-3}$ (magenta square and yellow circle) $\Phi=7 \times 10^{-3}$ (red squares and blue circles) and $\Phi=1.4 \times 10^{-2}$ (purple squares and green circles). The bottom panel shows that for flow experiments where both quantities have been measured, that $V^{*}$ is proportional to $D^{*}$. 


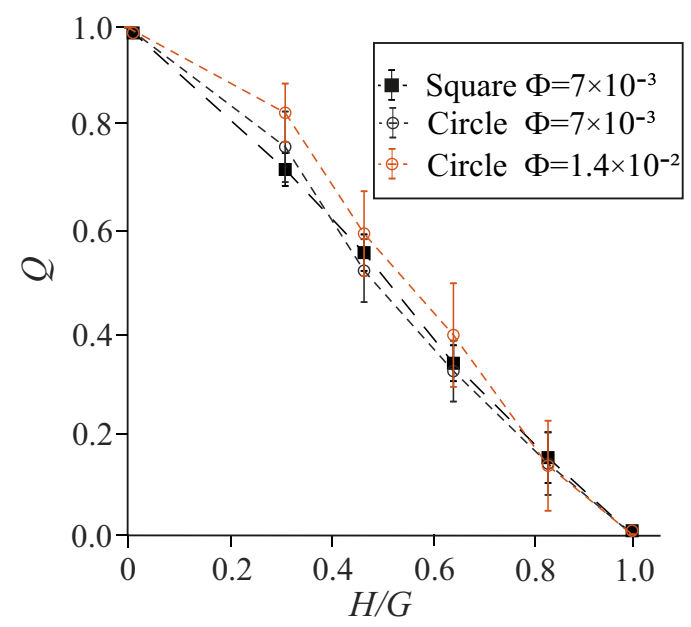

FIG. 15. Permeability factor as function of the height of the constriction $H$ that reduces locally the cell gap $G$, computed for various foam flow experiments with different liquid fractions of the foam and obstacle shapes.

Indeed, the occurrence of bubble rearrangements (T1s) prevents an infinite stretching of the bubbles. Furthermore, the threshold in bubble deformation for such yield transition is a function of the liquid fraction [13]: The larger the foam liquid fraction, the smaller the maximum deformation of the bubbles. Therefore, the maximal bubbles deformation and consequently the amplitude of the overshoot are set, on one hand, by the defect that stretches the bubbles and, on the other hand, limited by their plastic yielding, characterized by topological rearrangements through the swapping of neighboring bubbles.

Those processes lead to a complex flow pattern, which was already studied both experimentally and theoretically for nonpermeable obstacles [16], but with more complex features here, due to the partial permeability of the obstacle.

Our velocity measurements and specifically the measurements of the longitudinal velocity in the transverse direction to the mean flow, in the center of the cell, allow us to estimate a permeability factor of the model porous medium, quantifying the foam flow through and around the localized constriction. This permeability factor $Q$ is shown in Fig. 15 for experiments performed in various conditions (different obstacle shapes and liquid fractions) as a function of the obstacle height $H / G$. We observe a systematic quasilinear decrease of the permeability factor as the gap of the cell is locally reduced. The dispersion of our measurements does not allow to distinguish precisely an eventual impact of the experimental parameters on the cell permeability, which would deserve a thorough dedicated study. Nevertheless, we could extrapolate that for even wetter foams, the permeability factor could become zero for constriction height $H / G \simeq 0.85$. In these experimental conditions of a strong confinement, we could indeed observe that the bubbles of a wet foam do not flow through the constriction but simply around it, so that the constriction is acting as a nonpermeable obstacle.

To conclude, we have performed a thorough experimental study of a confined two-dimensional liquid foam (composed of a monolayered of bubbles) forced to flow within an inhomogeneous open fracture. Our model porous medium consists of a Hele-Shaw cell, with a single localized constriction, that reduces locally the cell gap and therefore decreases its permeability.

Analyzing the evolution of the texture of the flowing two-dimensional liquid foam (specifically the vertices and films of the bubbles, as well as their shape), we could compute the average steadystate velocity and bubble deformation fields of the foam, for various experiments, performed with 
different geometries of the obstacle (by changing its shape, size and height), as well as different amount of liquid within the formulated foams.

Our main result concerns the multipolar deformation and velocity fields with the strong fore-aft asymmetry of the foam flow-systematically observed for our various experiments-with a zone downstream the constriction, where the bubbles are strongly elongated in the mean direction of the flow and where the foam velocity can be much larger than the mean imposed driving velocity. While such symmetry breaking and velocity overshoot were already reported in previous studies for a liquid foam flowing around a nonpermeable obstacle $[12,13]$, we show here that such disturbance and recirculation can be modulated with the geometry of the obstacle and in particular, its height, which controls the local permeability of the cell. We, moreover, explain how our flow experiments confirm that the elastoplastic properties of the liquid foam, which are controlled by the foam liquid fraction, are responsible of this symmetry breaking of the flow deformation and velocity fields and, in particular, the overshoot downstream the constriction.

Finally, we could notice that the films of the bubbles could be pinned on the edges of the constriction, either at its inlet or outlet. This effect which is not present in the case of an obstacle filling completely the gap of the cell brings forward a capillary resistance to the flow and thus can impact the velocity field of the confined liquid foam. Further work is needed to clearly quantify such pinning and depinning of the bubble films and elucidate its consequences on the foam flow. Our first attempts to understand and predict the quasi-two-dimensional foam flow observed in our specific geometry, through and around a local constriction, based on the Durian "bubble" model $[35,36]$, could reveal the limits of such numerical approach to describe confined flows of dry foams. Indeed, we were not able to observe a clear asymmetry of the flow nor a significant velocity overshoot downstream the constriction.

We thank Benjamin Dollet, Renaud Toussaint, Lauren Rose, Juha Koivisto, and Evgeny Ermanyuk for enlightening discussions. This work is supported by ENS de Lyon, Grant No. 14.W03.31.0002 of the Russian Government, the French-Norwegian International Associated Laboratory, "D-FFRACT" (CNRS), and the Academy of Finland (Project No. 278367).

[1] I. Cantat, S. Cohen-Addad, F. Elias, F. Graner, R. Höhler, O. Pitois, F. Rouyer, and A. Saint-Jalmes, Foams (Oxford University Press, Oxford, 2013).

[2] S. Cohen-Addad, R. Höhler, and O. Pitois, Flow in foams and flowing foams, Annu. Rev. Fluid Mech. 45, 241 (2013).

[3] B. Dollet and C. Raufaste, Rheology of aqueous foams, C. R. Phys. 15, 731 (2014).

[4] P. Stevenson, Foam Engineering: Fundamentals and Applications (Wiley, New York, 2012).

[5] S. H. Talebian, R. Masoudi, T. I. M., and P. L. J. Zitha, Foam assisted $\mathrm{CO}_{2}$-EOR: A review of concept, challenges, and future prospects, J. Pet. Sci. Eng. 120, 202 (2014).

[6] A. R. Kovscek and H. J. Bertin, Foam mobility in heterogeneous porous media. I. Scaling concepts, Transp. Porous Media 52, 17 (2003).

[7] A. R. Kovscek and H. J. Bertin, Foam mobility in heterogeneous porous media. Experimental observations, Transp. Porous Media 52, 37 (2003).

[8] A. R. Kovscek and C. J. Radke, Fundamentals of foam transport in porous media, in foams: Fundamentals and applications in the petroleum industry, in Advances in Chemistry, edited by L. L. Schramm (American Chemical Society, Washington, DC, 1994), pp. 41-50.

[9] L. L. Schramm and F. Wassmuth, Foams: Basic principles, in Foams: Fundamentals and Applications in the Petroleum Industry, in Advances in Chemistry, edited by L. L. Schramm (American Chemical Society, Washington, DC, 1994), pp. 41-50. 
[10] L. W. Lake, Enhanced Oil Recovery (Prentice Hall, Upper Saddle River, NJ, 2010).

[11] W. R. Rossen, C. J. Van Duijn, Q. P. Nguyen, C. Shen, and A. K. Vikingstad, Injection strategies to overcome gravity segregation in simultaneous gas and water injection into homogeneous reservoirs, SPE J. 15, 76 (2010).

[12] B. Dollet and F. Graner, Two-dimensional flow of foam around a circular obstacle: Local measurements of elasticity, plasticity and flow, J. Fluid Mech. 585, 181 (2007).

[13] C. Raufaste, B. Dollet, S. Cox, Y. Jiang, and F. Graner, Yield drag in a two-dimensional foam flow around a circular obstacle: Effect of liquid fraction, Eur. Phys. J. E 23, 217 (2007).

[14] F. Graner, B. Dollet, C. Raufaste, and P. Marmottant, Discrete rearranging disordered patterns, part i: Robust statistical tools in two or three dimensions, Eur. Phys. J. E 25, 349 (2008).

[15] P. Marmottant, C. Raufaste, and F. Graner, Discrete rearranging disordered patterns, part II: 2D plasticity, elasticity and flow of a foam, Eur. Phys. J. E 25, 371 (2008).

[16] I. Cheddadi, P. Saramito, B. Dollet, C. Raufaste, and F. Graner, Understanding and predicting viscous, elastic, plastic flows, Eur. Phys. J. E 34, 1 (2011).

[17] B. Dollet, S. A. Jones, Y. Méheust, and I. Cantat, Influence of the elastic deformation of a foam on its mobility in channels of linearly varying width, Phys. Rev. E 90, 023006 (2014).

[18] B. Géraud, S. A. Jones, I. Cantat, B. Dollet, and Y. Méheust, The flow of a foam in a two-dimensional porous medium, Water Resour. Res. 52, 773 (2016).

[19] W. R. Rossen, Theory of mobilization pressure gradient of flowing foams in porous media. I. Incompressible foam, J. Colloid Interface Sci. 136, 1 (1990).

[20] Q. Xu and W. R. Rossen, Effective viscosity of foam in periodically constricted tubes, Colloids Surf. A 216, 175 (2003).

[21] S. Cox, S. Neethling, W. R. Rossen, W. Schleifenbaum, P. Schmidt-Wellenburg, and J. J. Cilliers, A theory of the effective yield stress of foam in porous media: The motion of a soap film traversing a three-dimensional pore, Colloids Surf. A 245, 143 (2004).

[22] K. Ma, R. Liontas, C. A. Conn, G. J. Hirasaki, and S. L. Biswal, Visualization of improved sweep with foam in heterogeneous porous media using microfluidics, Soft Matter 8, 10669 (2012).

[23] R. Liontas, K. Ma, G. J. Hirasaki, and S. L. Biswal, Neighbor-induced bubble pinch-off: Novel mechanisms of in situ foam generation in microfluidic channels, Soft Matter 9, 10971 (2013).

[24] B. Géraud, Y. Méheust, I. Cantat, and B. Dollet, Lamella Division in a Foam Flowing Through a Two-Dimensional Porous Medium: A Model Fragmentation Process, Phys. Rev. Lett. 118, 098003 (2017).

[25] T. Chevalier, J. Koivisto, N. Shmakova, M. J. Alava, A. Puisto, C. Raufaste, and S. Santucci, Foam flows through a local constriction, J. Phys. Conf. 925, 1 (2017).

[26] C. Raufaste, A. Foulon, and B. Dollet, Dissipation in quasi-two-dimensional flowing foams, Phys Fluids 21, 053102 (2009).

[27] See Supplemental Material at http://link.aps.org/supplemental/10.1103/PhysRevFluids.xx.xxxxxx for a typical recorded movie of a liquid foam invading our inhomogeneous cell.

[28] W. Thielicke and E. Stamhuis, Pivlab - towards user-friendly, affordable and accurate digital particle image velocimetry in matlab, J. Open Res. Softw. 2, e30 (2014).

[29] C. Raufaste, B. Dollet, S. Santucci, and R. Mokso, Three-dimensional foam flow resolved by fast X-ray tomographic microscopy, Europhys. Lett. 111, 38004 (2015).

[30] K. Mader, R. Mokso, C. Raufaste, B. Dollet, S. Santucci, J. Lambert, and M. Stampanoni, Quantitative 3D characterization of cellular materials: Segmentation and morphology of foam, Colloids Surf. A 415, 230 (2012).

[31] O. Hassager, Negative wake behind bubbles in non-newtonian fluids, Nature 279, 402 (1979).

[32] N. Roquet and P. Saramito, An adaptive finite element method for bingham fluid flows around a cylinder, Comput. Methods Appl. Mech. Eng. 192, 3317 (2003).

[33] A. Saint-Jalmes and D. Durian, Vanishing elasticity for wet foams: Equivalence with emulsions and role of polydispersity, J Rheol. 43, 1411 (1999). 
[34] M. Asipauskas, M. Aubouy, J. A. Glazier, F. Graner, and Y. Jiang, A texture tensor to quantify deformations: The example of two-dimensional flowing foams, Granul. Matter 5, 71 (2003).

[35] D. J. Durian, Foam mechanics at the bubble scale, Phys. Rev. Lett. 75, 4780 (1995).

[36] V. J. Langlois, The two-dimensional flow of a foam through a constriction: Insights from the bubble model, J. Rheol. 58, 799 (2014). 